\title{
Debugging and Application Test for Integrated Navigation System
}

\author{
Zhi Li \\ Computer Teaching and Research Section \\ Chongqing Vocational College of \\ Architectural Engineering \\ Chongqing,China \\ 1002030665@qq.com
}

\author{
Jinjiang Liu \\ Chongqing Aerospace New Century \\ Satellite Application Technology Co.,Ltd \\ Chongqing,China \\ 525572284@qq.com
}

\begin{abstract}
We integrated the reality and the development of the navigation technology at home and abroad, considered the operating feature of underwater wheeled mining vehicles for mines and their poor working conditions, applied the laser gyro inertial autonomous navigation system advanced at home and abroad during the cooperation between the college and enterprises, and thus have designed the underwater integrated navigation system. The application test shows: The integrated navigation system researched in this paper enjoys a proper design scheme, achieves the expected navigation accuracy and primarily overcomes the positioning error accumulated over time.
\end{abstract}

Keywords-Strap down Inertial Navigation System (SINS), C language, MS-DOS Operating System, Accelerometer, Milemeter, Integrated Navigation

\section{Debugging OF INTEGRATEd NAVIGATION System}

The integrated navigation system is composed of the laser gyro SINS, the temperature sensor data collection, the integrated navigation software, the milemeter, the data processing computer, and the mounting transition plate, cables, etc. The system structure is shown in Fig. 1.

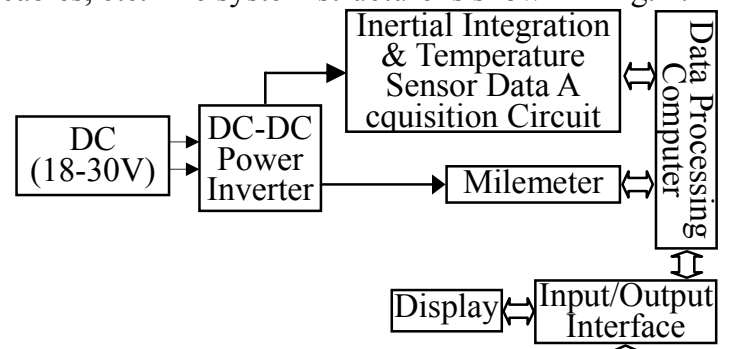

Fig. 1 The System Structure

1. Hardware Debugging

The self-made special debugging plate debugs the hardware of the system. The other measuring instruments required by the debugging are shown in Table 1:
TABLE I . MEASURING INSTRUMENTS UTILIZED

\begin{tabular}{|c|c|c|}
\hline Description & Model & Key Indicators \\
\hline $\begin{array}{c}\text { DC } \\
\text { Stabilized } \\
\text { Power } \\
\text { Supply } \\
\end{array}$ & $\begin{array}{l}\text { GPS- } \\
3303 C\end{array}$ & $\begin{array}{l}\text { Voltage: } 0 \sim 30 \mathrm{~V} \\
\text { Max. Supply Current: } 10 \mathrm{~A} \\
\text { Voltage Accuracy: } \pm 1 \% \mathrm{~V} \\
\text { Current Accuracy: } \pm 1 \% \mathrm{~A}\end{array}$ \\
\hline $\begin{array}{c}\text { Digital } \\
\text { Multimeter }\end{array}$ & 34401A & $\begin{array}{l}\text { Voltage: } 1 \mathrm{mV} \sim 1000 \mathrm{~V} \\
\text { Voltage Accuracy: } 0.0008 \% \\
\text { Current: } 1 \mathrm{nA} \sim 100 \mathrm{~A} \\
\text { Current Sensitivity: } 1 \mathrm{pa} \\
\text { Resistance: } 10 \Omega \sim 1000 \Omega \\
\text { Resistance Sensitivity: } 10 \mu \Omega\end{array}$ \\
\hline Oscilloscope & TDS1002 & $\begin{array}{l}\text { Frequency Bandwidth: } 60 \mathrm{MHz} \\
\text { Transient Response Time:5.8ns }\end{array}$ \\
\hline
\end{tabular}

(1) Connection: First, switch on the DC stabilized power supply, and turn the two routes of the output values of the power supply to $24 \mathrm{~V}$, right after that, power off. Second, connect the power plug on the inertial navigation system to the DC power supply. Finally, connect the port output plug to be debugged to $\mathrm{PC}$ and meanwhile, extract the signal output plug of the self-testing plate.

(2) Debugging: Power on, measure the output of the debugging circuit board with the oscilloscope and check the output waveform. If there is no signal output or the output signal is abnormal, there must be some failures; otherwise, the test is completed.

2. Software Debugging

When debugging the system hardware, debug the system software.

The system software is programmed in C language and runs in the computer DOS system, with the main process flow as shown in Fig. 2 and the system initial alignment process flow as shown in Fig. 3. 


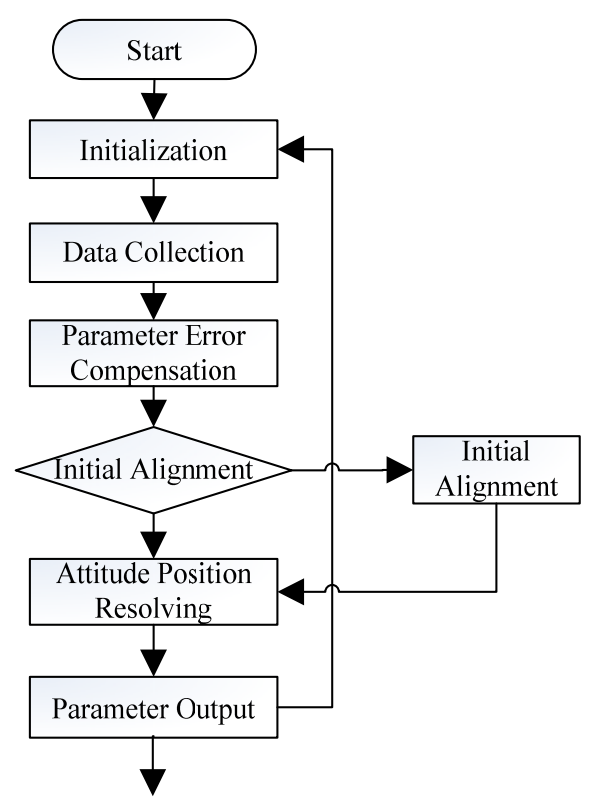

Fig. 2 System Main Process Flow

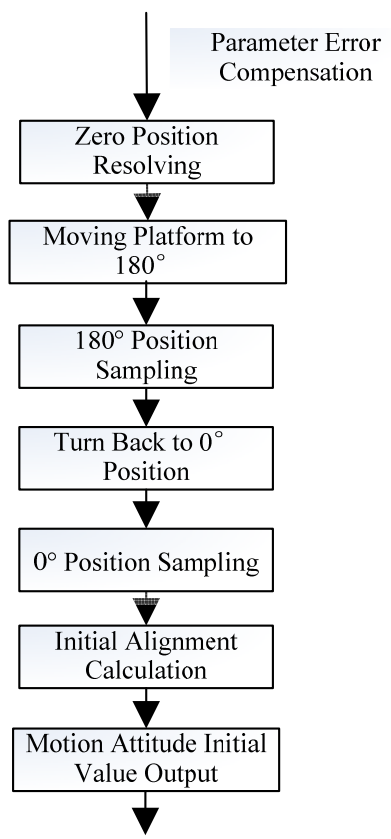

Fig. 3 System Initial Alignment Process Flow

3. Error Parameter Calibration of Gyro and Accelerometer

The inertial instruments include the gyro and the accelerometer, which are the most critical components in the SINS hardware. The accelerometer senses the accelerated speed of the carrier, while the gyro tests the attitude angle rate. Their performances are closely related to a series of performance indicators of the system. The errors of the inertial instruments are the main elements influencing the accuracy of the inertial system.
The gyro is required to not only measure the tiny angular displacement and provide clear response signal meeting the resolution requirement, but also limit its drift error within a range as small as possible. The drift error of the gyro generally includes the constant drift, the certainty drift, the random drift, etc. The constant drift can be determined by experimental measurement before the gyro is mounted in the system and can be adjusted. The certainty drift includes the static error and the dynamic error; the former is caused by the linear motion of the carrier, while the latter, by the angular motion. The certainty drift may build the gyro static error model and dynamic error model by the off-line test and program the error compensation software and install in the navigation computer. The real-time compensation can be carried out on the basis of the linear motion and the angular motion of the carrier. The scale factor error and the installation error of the gyro can also be tested off line, modeled and compensated.

The accelerometer is also required to have high resolution which is able to clearly and precisely reflect the accelerated speed ranged from a span as wide as possible, and provide corresponding signal. Meanwhile, it must have stable and as-small-as possible zero error. The linear motion and the angular motion of the carrier will cause the error of the accelerometer as well. Besides, the accelerometer also has installation error and scale factor error, all of which could be compensated by modeling. Therefore, in order to improve the accuracy of the inertial instrument, it is necessary to conduct the calibration test to inertial elements. Our test adopts the separate calibration method, with the calibration result as shown in Table 2:

TABLE II. CALIBRATION RESULTS

\begin{tabular}{|c|c|c|c|c|c|c|}
\hline Parameters & Sgox & Sgoy & Sgoz & Soyz & Sozy & Soxz \\
\hline Results & 1.2206 & -1.2246 & -1.2245 & -50.54699 & 2.32135 & -49.28388 \\
\hline Parameters & $\triangle_{\text {ozx }}$ & $\triangle_{\text {oxy }}$ & $\triangle_{\text {oyx }}$ & & & \\
\hline Results & -2.32135 & -0.84239 & -0.89349 & & & \\
\hline
\end{tabular}

4. Initial Alignment Test of Inertial Integration

The alignment test is carried out with the employment of the calibration result of the error parameter of the gyro and the accelerometer, which is realized on the 902C-2 type double-spindle position rate rotary table. The test steps are as follows:

(1) Place the inertial integration primarily horizontally, initially align and turn the course near $0^{\circ}$ after the alignment;

(2) Keep the inertial integration still, align repeatedly for six times, record the data, and after recording the sixth alignment, turn the inertial integration under the state of navigation to make the course angle near $90^{\circ}$ and record the data;

(3) Keep the inertial integration still, align repeatedly for six times, record the data, and after recording the sixth alignment, turn the inertial integration under the state of navigation to make the course angle near $180^{\circ}$ and record the data;

(4) Keep the inertial integration still, align repeatedly for six times, record the data, and after recording the sixth 
alignment, turn the inertial integration under the state of navigation to make the course angle near $270^{\circ}$ and record the data;

(5) Keep the inertial integration still, align repeatedly for six times, record the data, and after recording the sixth alignment, turn the inertial integration under the state of navigation to make the course angle near $0^{\circ}$ and record the data;

(6) Keep the inertial integration still, initially align again, record the data, and after the recording, turn the inertial integration under the state of navigation to make the pitch angle near $5^{\circ}$, and record the data;

(7) Keep the inertial integration still, initially align again, record the data, and after the recording, turn the inertial integration under the state of navigation to make the pitch angle near $-5^{\circ}$ and record the data;

(8) Keep the inertial integration still, initially align again, record the data, and after the recording, turn the inertial integration under the state of navigation to make the pitch angle near $0^{\circ}$ and the roll angle near $5^{\circ}$, and record the data;

(9) Keep the inertial integration still, initially align again, record the data, and after the recording, turn the inertial integration under the state of navigation to make the roll angle near $-5^{\circ}$, and record the data

5. System Integration and Test

After the aforesaid debugging, confirm that all parts of the integrated navigation system are running normally and in good condition. Connect and assemble all parts according to the system structure in Fig. 5.1; after the completion of the assembly, debug the system.

Instruments required by the debugging: a computer and the same instruments as those used in the hardware debugging.

(1) Connection: First, switch on the DC stabilized power supply, and turn the two routes of the output values of the power supply to $24 \mathrm{~V}$, and after that, power off; second, connect the power interface of the system to the DC power supply; finally, connect the input/output interface of the system to the computer.

(2) Debugging: Power on and start the computer. Make the integrated navigation system continuously run, and manually rotate the main spindle of the milemeter and observe the attitude angle of the system and the mile meter data gained by the computer.

6. Site Debugging

Debug the system on the site of the provider of the system. The procedures are roughly the same as those in the section 5 . The primary focus is on testing the accuracy of the system.

\section{APPLICATION TEST AND ITS RESULT ANALYSIS}

After the completion of the site debugging of the system, carry out the application test of the system --- realizing the underwater position and azimuth determining.

The underwater test employs the navigating wheeled test car equipped with integrated navigation system and a sign post on its top. The environment is a pool of $200 \mathrm{~m} 2$. The whole journey of the test car is about $20 \mathrm{~min}$ and the speed is $0.1 \mathrm{~m} / \mathrm{s}$. With the center of the east position of the pool being the starting point and the east position being the benchmark, the position data of the test car are recorded every $5 \mathrm{~m}$ it travels, with the data recorded in Table 3. In Table 3: The data in the team a are the position coordinate data collected by the integrated navigation system; and the team $b$, the position coordinate data collected by the sign post on the top of the test car.

TABLE III. EAST \& NORTH POSITION COORDINATES DATA COMPARISON TABLE

\begin{tabular}{|c|c|c|c|c|c|c|c|}
\hline $1 \mathrm{a}$ & $(-5,3.01)$ & $5 \mathrm{a}$ & $(-15,30.08)$ & $9 \mathrm{a}$ & $(5,29.72)$ & $13 \mathrm{a}$ & $(15,9.01)$ \\
\hline 1b & $(-5,3.02)$ & $5 \mathrm{~b}$ & $(-15,30.42)$ & $9 \mathrm{~b}$ & $(5,29.63)$ & $13 \mathrm{~b}$ & $(15,8.79)$ \\
\hline 2a & $(-10,5.11)$ & $6 \mathrm{a}$ & $(-10,30.51)$ & $10 \mathrm{a}$ & $(10,30.12)$ & $14 \mathrm{a}$ & $(10,4.33)$ \\
\hline 2b & $(-10,5.31)$ & $6 \mathrm{~b}$ & $(-10,30.32)$ & $10 \mathrm{~b}$ & $(10,30.07)$ & $14 \mathrm{~b}$ & $(10,4.12)$ \\
\hline 3a & $(-15,8.12)$ & $7 \mathrm{a}$ & $(-5,30.11)$ & $11 \mathrm{a}$ & $(15,27.01)$ & $15 \mathrm{a}$ & $(5,2.01)$ \\
\hline 3b & $(-15,8.49)$ & $7 \mathrm{~b}$ & $(-5,30.23)$ & $11 \mathrm{~b}$ & $(15,27.58)$ & $15 \mathrm{~b}$ & $(5,2.38)$ \\
\hline 4a & $(-20,14.92)$ & $8 \mathrm{a}$ & $(-0,30.13)$ & $12 \mathrm{a}$ & $(20,13.11)$ & $16 \mathrm{a}$ & $(0,0.05)$ \\
\hline 4b & $(-20,14.47)$ & $8 \mathrm{~b}$ & $(-0,30.17)$ & $12 \mathrm{~b}$ & $(20,13.73)$ & $16 \mathrm{~b}$ & $(0,0.17)$ \\
\hline
\end{tabular}

The navigation trajectory chart drawn based on the data in the above-mentioned table is shown as Fig. 4:

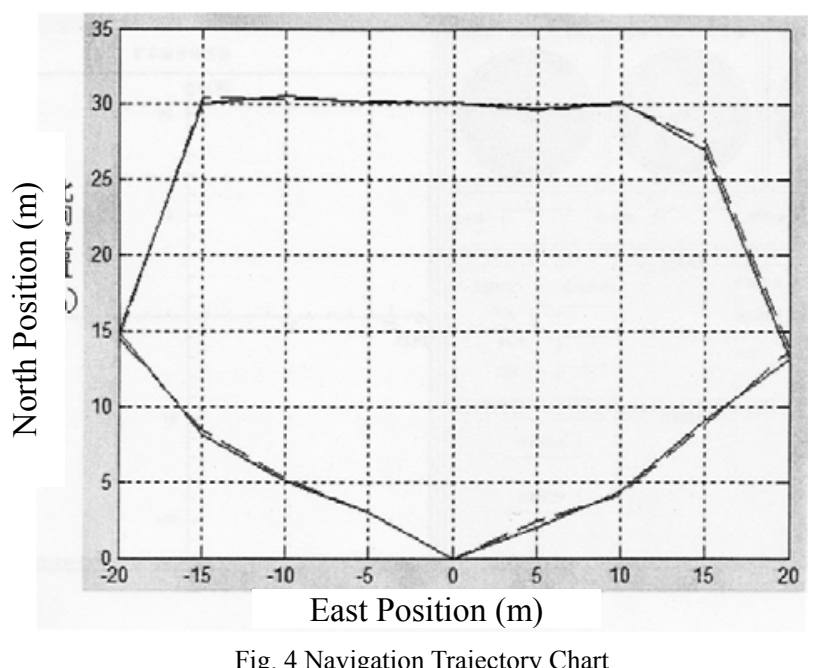

In Fig. 4, the solid line is drawn according to the test car position data collected by the integrated navigation system, while the dashed line is based on the data measured by the sign post on the top of the test car.

TABle IV. North Position ERror Data TABle

\begin{tabular}{|c|c|c|c|c|c|c|c|}
\hline 0.01 & 0.37 & 0.34 & 0.12 & -0.09 & 0.57 & -0.22 & 0.37 \\
\hline 0.2 & -0.45 & -0.19 & 0.04 & -0.05 & 0.62 & -0.21 & 0.12 \\
\hline
\end{tabular}

Based on the data in Table 4, the maximum north position error of the integrated navigation system is $0.62 \mathrm{~m}$, and therefore we thusly conclude that the milemeter can be used to assist the navigation of SINS so that a relatively high level of positioning accuracy of the system could be reached.

\section{CONCLUSION}

SINS/OD integrated navigation system can solve the weakness of the SINS navigation position error accumulated remarkably over time. The integrated navigation system 
enjoys a better positioning performance and the stronger anti-interference capacity and autonomy. Therefore, the said autonomous integrated navigation system is to some extent practical for the navigation of the underwater carrier.

\section{REFERENCES}

[1] Xin Chang'an. VC++ Programming Techniques and Difficulties Analysis [M]. Tsinghua University Press: 40-48.
[2] Yang Shuying. VC++ Picture Processing Programming [M]. Tsinghua University Press: 50-62.

[3] Zhao Zhong, Ma Rong and Li Ruigong. Development of an SINS with Low Cost [J]. Projectile and Guidance Journal. 2000.

[4] Su Jinming and Ruan Shenyong. MATLAB6.1 Practical Guidance [M]. Publishing House of Electronics Industry. 2002:28-36.

[5] Zhang Yihua. Master MATLAB 5 [M]. Tsinghua University Press. 1999 